\title{
Estresse Psicológico e Enfrentamento em Mulheres Com e Sem Câncer ${ }^{1}$
}

\author{
Carmen Maria Bueno Neme ${ }^{2}$ \\ Universidade Estadual Paulista (Bauru) \\ Marilda Emmanuel Novaes Lipp \\ Pontificia Universidade de Campinas
}

\begin{abstract}
RESUMO - Considerando o alto índice de mortes de mulheres por cânceres de mama, útero e ovários e resultados de pesquisas indicando relações entre estresse, enfrentamento e doenças como as oncológicas, este estudo investigou, em 30 mulheres com e 30 mulheres sem câncer, a ocorrência de estresse em suas histórias prévias, a importância atribuída ao mesmo e a avaliação de sua superação, nas áreas de saúde, social/trabalho e familiar. Os resultados indicaram relações entre os modos de avaliar e enfrentar o estresse e o adoecimento, sugerindo que padrões mais otimistas e diretos de lidar com o estresse favoreceram a redução de seu impacto no equilíbrio psicofisiológico. Dada a exposição crescente da mulher ao estresse, indica-se a relevância de programas psicoeducativos redutores de seu impacto na população feminina.
\end{abstract}

Palavras-chave: psico-oncologia; cânceres femininos; estresse; enfrentamento.

\section{Psychological Stress and Coping in Women With and Without Cancer}

\begin{abstract}
Considering the high death rate of women for breast, uterus and ovary cancers, and research findings indicating links between stress, coping and oncologic diseases, this study investigated, in 30 women with and 30 women without cancer, the occurrence of stress in their previous history as well as the importance attached to the it, and their evaluation of its resilience in the health care, social/work and family areas. The results indicated links between the ways of evaluating and facing stressful situations and the illness condition, suggesting that more optimistic and direct patterns of dealing with stress can reduce its impact on human psychological/physiological balance. Given the increasing exposure of women to stress, psychological and educational programs for reducing the impact of stress on the female population are indicated.
\end{abstract}

Keywords: psycho-oncology; female cancers; stress; coping.

Os avanços recentes obtidos nas técnicas diagnósticas, terapêuticas e medicamentosas em oncologia pouco mudaram a visão do câncer como uma das doenças mais ameaçadoras e mortais. Atingindo amplas parcelas da população, ao lado das doenças cardiovasculares e dos óbitos por acidente, o câncer encontra-se entre as três principais causas de morte no Brasil e no mundo, segundo dados do Instituto Nacional do Câncer (INCA, 2008).

Entre as mulheres, os cânceres de mama figuram entre as primeiras causas de mortalidade. Somando-se aos de ovários e de útero, os cânceres femininos levam à morte milhares de mulheres por ano no Brasil e no mundo. Embora possam ser prevenidos e eficientemente tratados quando diagnosticados precocemente, ainda representam uma grande preocupação em nível de saúde pública (INCA, 2004, 2008; Neme, 2005; Neme, Soliva \& Ribeiro, 2003).

Os cânceres de colo uterino e de ovários também exercem importante papel na morbidade e mortalidade de mulheres, com repercussões do ponto de vista da saúde pública e significativo impacto na qualidade de vida, sexualidade e

1 Este trabalho é parte do projeto de pós-doutoramento da primeira autora, desenvolvido no Laboratório de Pesquisas Psicofisiológicas do Stress (PUC-Campinas), com Bolsa de Pós-Doutorado do CNPq.

2 Endereço para correspondência: Rua Vivaldo Guimarães, 234, Jardim Estoril. Bauru, SP. CEP 17016- 070. Fone: (14) 322419 03. E-mail: cmneme@gmail.com. funcionamento psicossocial da população afetada. Os cânceres de mama e de colo de útero são os de maior incidência entre as mulheres brasileiras e a resposta aos tratamentos depende, assim como nos demais casos de neoplasia maligna, do estadiamento clínico inicial da doença (Thuler \& Mendonça, 2005).

No Brasil, poucos estudos analisaram as tendências temporais do estadiamento dos casos de câncer. Dados obtidos por Thuler e Mendonça (2005) em Centros de Alta Complexidade cadastrados no Ministério da Saúde e com atendimento pelo Sistema Único de Saúde (SUS), demonstraram que na última década houve redução no percentual de casos de câncer de mama e de colo do útero em estadio avançado, indicando que em algumas regiões houve melhora em sua detecção precoce. Por outro lado, Trufelli e cols. (2008), em um estudo retrospectivo, analisaram o atraso no diagnóstico e no tratamento de 68 casos de câncer de mama em hospital no Estado de São Paulo e concluíram que mulheres tratadas em serviço público sofrem atrasos importantes, principalmente no período de diagnóstico. Entre os intervalos analisados, o maior atraso ocorreu entre a suspeita mamográfica de câncer e a biópsia. A demora para iniciar o tratamento foi significativamente maior em mulheres com câncer mais avançado, comparativamente àquelas com câncer de estadio inicial. Os autores alertam para a necessidade de diminuir o intervalo entre a mamografia e a biópsia de lesões suspeitas. 
Quanto à detecção precoce dos cânceres de útero, são poucos os estudos sobre cobertura do exame de Papanicolaou no Brasil. Martins, Thuler e Valente (2005) observaram que a maioria dos estudos se concentra nas regiões sul e sudeste e que há pouca padronização metodológica em relação à amostragem e ao perfil das mulheres pesquisadas, dificultando a comparação de resultados. Apesar dessas dificuldades, os autores constataram uma tendência de aumento no percentual de mulheres que foram submetidas a pelo menos um exame de Papanicolaou nos últimos anos. As mulheres não submetidas ao exame tinham baixo nível de escolaridade, baixa renda familiar e eram mais jovens. Os resultados apontaram desigualdades regionais na cobertura do exame na população feminina brasileira, indicando a necessidade de intervenção junto aos fatores identificados. Estudos realizados por Neme $(1999,2005)$ com 130 pacientes oncológicos também identificaram que os maiores níveis de gravidade do câncer no diagnóstico inicial relacionaram-se positivamente à baixa escolaridade, tanto entre homens como entre mulheres, alertando sobre a necessidade de intervenções para orientar a população quanto aos benefícios dos exames preventivos, bem como a urgência de melhoria nos serviços públicos de prevenção do câncer.

No caso das neoplasias femininas, outro fator que dificulta a detecção precoce é a dificuldades das mulheres em se submeter aos exames preventivos. Estudo realizado por Duavy, Batista, Bessa e Santos (2007) mostrou que embora o câncer pélvico uterino seja uma doença crônica degenerativa das mais temidas, a mulher geralmente procura fazer o exame apenas quando tem sintomas e vê o procedimento com apreensão e temor pela possibilidade de um diagnóstico positivo de câncer. A mulher costuma sentir-se constrangida pela exposição do corpo, principalmente quando o profissional é do sexo masculino, o que resulta em resistência em se submeter aos exames preventivos.

Muitas pesquisas indicam o impacto das doenças oncológicas femininas na vida familiar, sexual, afetiva e psicossocial da mulher, demonstrando o caráter ameaçador, estigmatizante e permeado de inúmeras dificuldades e preconceitos que a doença possui (Bernardo, Lorenzato, Figueiroa \& Kitoko, 2007; Neme \& Kato, 2003).

Ao revisar amplamente os fatores psiquiátricos envolvendo o câncer de mama, Cantinelli e cols. (2006) ressaltaram seu impacto na saúde mental devido às possibilidades cirúrgicas e aos seus efeitos sobre a imagem corporal, autoestima e sexualidade, e aos tratamentos sistêmicos e às suas consequências físicas, tais como fadiga, náuseas e vômitos.

Quanto mais grave a condição da mulher com câncer, maiores são as perdas físicas, psicológicas e os riscos de co-morbidades, gerando dificuldades crescentes para o enfrentamento da doença, dos tratamentos e das mudanças sociais, econômicas e de rotina de vida individual e familiar. A vida da mulher fica comprometida e a possibilidade de morte é mais concreta, dependendo da gravidade da doença no início do tratamento (Cantinelli \& cols., 2006; Maluf, Mori \& Barros, 2005).

\section{Estresse, Enfrentamento e o Câncer na Mulher}

O conceito de estresse ou Síndrome Geral de Adaptação (GAS) foi desenvolvido por Selye em 1936, definindo um amplo processo de interações indivíduo-ambiente, que acarreta profundas modificações fisiológicas necessárias à adaptação frente a eventos potencialmente nocivos ao indivíduo (Selye, 1976).

Ao pesquisar o papel da mediação cognitivo-afetiva na situação de estresse e enfrentamento, Lazarus e Folkman (1986), Lipp (2001) e Lipp, Frare e Santos (2007) demonstraram o importante papel de variáveis individuais e culturais no desencadeamento do estresse e em seus efeitos. O processo de enfrentamento do estresse dependerá, em grande parte, dos recursos sociais e psicológicos do indivíduo. Esse processo manifesta-se de modo diferente em diferentes indivíduos e pode variar, no mesmo indivíduo, em diversos momentos de seu ciclo vital, em situações ou contingências diversas, dependendo de como é percebido e avaliado (Lipp, Pereira \& Sadir, 2005; Neme, 2005; Rowland, 1990).

Rápidas e extensas mudanças têm ocorrido na pós-modernidade nas áreas da saúde e da tecnologia e nas organizações política, econômica, social e familiar, além de profundas alterações na organização mundial e das micro-organizações sociais, trazendo a necessidade de contínuas adaptações e o aumento das chamadas doenças de adaptação (Giovanetti, 2002; Lipp, 2001; Neuber \& cols., 2007).

Embora homens e mulheres pareçam ser igualmente afetados pelo estresse, as mulheres têm sido sobrecarregadas pela multiplicidade de papéis sociais e familiares que atualmente assumem, estando mais vulneráveis a doenças caracteristicamente "masculinas", como as cardiopatias e as doenças vasculares. Aumentam as patologias específicas femininas como a amenorréia, a síndrome pré-menstrual, a infertilidade, a depressão pós-parto, o vaginismo e outras doenças hormônio-dependentes, tais como as produzidas em situações de estresse pela diminuição da progesterona, acarretando desequilíbrios orgânicos consideráveis e disfunções psiconeuroendócrinas e imunológicas gerais (Calais, 2003; Neuber \& cols., 2007). Alguns estudos indicam que a mulher apresenta maior nível de estresse psicológico e maior sensibilidade emotiva do que os homens, aspectos especialmente vinculados a seus papéis familiares e conjugais (Calais, Andrade \& Lipp, 2007; Neuber \& cols., 2007).

Os eventos de estresse potencialmente mais patogênicos são os representados por pequenos acontecimentos repetidos por longos períodos de tempo e experimentados quase que imperceptivelmente, ao invés das ocorrências extraordinárias ocasionais, para as quais são mais facilmente mobilizados recursos e estratégias para superá-las (Lazarus \& Folkman, 1986; Lipp, 2007; Lipp \& cols., 2007; Lipp, Justo \& Melo Gomes, 2006; Neme, 2005). De acordo com Neme e cols. (2003), as maiores fontes de estresse apontadas pelas participantes da pesquisa, mulheres com câncer, estavam ligadas a relações familiares e a problemas nessa área da vida, corroborando dados obtidos por Neme (1999) com pacientes de ambos os sexos. Os problemas familiares relatados foram compreendidos como fontes prolongadas de estresse, cujo 
enfrentamento exige esforços contínuos e repetidos no cotidiano, aumentando seu potencial estressogênico.

De acordo com os modos individuais de enfrentamento, aspectos de personalidade, pré-existência ou não de algum transtorno como o depressivo ou ansioso, ou ainda, de tendências ou padrões afetivo-comportamentais pessoais, familiares e socioculturais, o papel das condições pessoais na avaliação do estresse poderá ser preponderante em relação ao conjunto dos demais fatores envolvidos no processo de enfrentamento (Carver \& Scheier, 1994; Lipp \& cols., 2007; Neme, 2005). A manutenção de sentimentos e emoções que resultam em mal-estar ou contrariedade em decorrência de eventos de vida que exigem superação e readaptações constitui-se contínua fonte interna de estresse, prolongando e agravando seus inevitáveis efeitos psicofisiológicos sobre os diferentes sistemas orgânicos e predispondo o indivíduo a diversos tipos de doenças, especialmente as que se relacionam ao funcionamento imunológico, incluindo o câncer (Lipp, 2005; Neme \& cols., 2003)

Pesquisas sobre mulheres com câncer mostram que essas revelam, em sua história de vida, vivências associadas a acontecimentos traumáticos, eventos de vida negativos, dificuldades na regulação e elaboração de conflitos, lutos complicados e a manutenção de sentimentos geradores de mal-estar como tristeza, raiva e mágoas (Filgueiras \& cols., 2007; Marszal-Wisniewska \& Zakrzewska, 2004; Neuber \& cols., 2007).

Estados emocionais positivos ou negativos têm sido pesquisados, buscando-se suas possíveis relações com o câncer e outras doenças. De Moor e cols. (2006) investigaram, em 90 mulheres com câncer de ovário e com prescrição de quimioterapia, se o otimismo situacional ou disposicional era capaz de proteger contra o estresse e melhorar a qualidade de vida. Avaliaram também se o otimismo era capaz de prever o decréscimo nos níveis do antígeno de câncer (CA 125) durante o tratamento. As mensurações foram realizadas por exames médicos no início e no final dos ciclos de quimioterapia. A amplitude dos dados coletados possibilitou o estabelecimento de conexões entre as variáveis psicossociais e as mensurações objetivas da progressão da doença, mostrando a relevância do otimismo situacional na redução do CA 125 e do otimismo disposicional na promoção da saúde e qualidade de vida das pacientes.

A depressão, frequentemente associada a alterações fisiológicas também é apontada como fator que predispõe o organismo a várias enfermidades, entre elas, o câncer. Muitos estudos têm procurado relacionar depressão e alterações dos sistemas imune e hormonal (Bauer, Gauer \& Nardi, 1993). Ao investigar possíveis efeitos orgânicos da depressão, Nunes e cols. (2002) realizaram mensurações imunes e hormonais em 40 pacientes adultos com depressão, de moderada a muito severa, e não medicados. Os resultados revelaram uma redução significativa de albumina e elevação do $\alpha-1$, $\alpha-2$ e $\beta$-globulinas e do receptor IL-2 solúvel nos pacientes com depressão, comparativamente ao grupo controle. Esses resultados confirmam o distúrbio imunológico em pacientes depressivos, embora os autores tenham indicado a necessidade de novas investigações, dada a possível interação de outras variáveis.
No estudo da gênese do câncer, a literatura indica a necessidade de estudos mais detalhados acerca das variáveis subjetivas pessoais e das situações e contexto do estresse na história dos sujeitos afetados. Importante estudo de revisão sobre estresse e câncer, realizado por Carleial (1981), concluiu pela necessidade de se pesquisar a história de vivências estressantes no período de um a 10 anos antes da manifestação da doença. Determinados eventos ocorridos há anos poderão ser vivenciados como recentes, como é o caso de certas formas de luto complicado, dificultando ou mesmo impedindo sua superação (Neme, 2005; Neme \& cols., 2003).

Dada a multiplicidade de fatores e condições possivelmente relacionados ao câncer, sua identificação etiológica ainda é um problema aberto para a pesquisa. Estudos no campo da Psiconeuroimunologia têm tentado clarificar os aspectos orgânico-celulares relacionados ao desenvolvimento de neoplasias e de outras doenças associadas ao funcionamento imune e à intermediação de emoções, sentimentos e cognições (Solomon, 1993). Ao realizar uma revisão bibliográfica de estudos em psicoimunologia, Moreira (1994) apontou a prevalência de indicadores da atuação de fatores emocionais no aparecimento e desenvolvimento de processos cancerosos nos estudos revisados. Segundo Moreira, entre os pacientes portadores de neoplasias malignas estudados, não foi encontrado um único caso em que um fator emocional de estresse não estivesse associado à origem da doença. Com base nos resultados das pesquisas revisadas, Moreira ressaltou que os estados afetivos (particularmente a depressão), além de fatores psicossociais, relacionam-se ao aparecimento do câncer, dada sua influência no sistema imunológico.

Amorim (1999) estudou o efeito do relaxamento no sistema imunológico, comparando dois grupos de mulheres com cânceres de mama sem metástases à distância e submetidas a cirurgias. Esse autor utilizou a atividade das células NK (Natural Killer) como parâmetro imunológico e mensurou o padrão de comportamento para estresse e coping, a ansiedade e a ressonância corporal. Seus resultados demonstraram estatisticamente que o relaxamento modulou e aumentou a atividade NK do sistema imune.

Constata-se crescente interesse pela compreensão da relação entre funcionamento mental, imunidade e condição da doença. Na maioria das vezes, esse interesse se manifesta em estudos estabelecendo associações entre circunstâncias de vida, estados afetivos e aspectos de personalidade e adoecimento. A literatura apresenta associações entre posturas defensivas particulares, envolvendo a supressão de afetos, principalmente agressivos, a evitação de conflitos e o desenvolvimento das neoplasias malignas (Carvalho, 1994; Dorian \& Garfinkel, 1987; Levin \& Kissane, 2006; Neme, 2005; Temoshok, 1987).

Observa-se, também, significativo aumento de interesse pela investigação de aspectos relacionados ao enfrentamento e à qualidade de vida em mulheres com cânceres de mama e ginecológicos, demonstrando a relevância do impacto dessas neoplasias malignas na vida da mulher e a necessidade de assistência multiprofissional a pacientes e familiares, desde o diagnóstico até o controle da doença ou terminalidade (Bernardo \& cols., 2007; Maluf \& cols., 2005; Müller, 
Hoffmann \& Fleck, 2006; Neme, 2005; Okamura, Yamawaki, Akechi, Taniguchi \& Uchitomi, 2005; Venâncio, 2004).

Considerando o alto índice de mortes de mulheres por cânceres de mama, útero e ovários, a relevância da prevenção e os resultados de pesquisas indicando as relações de influência entre o estresse, os modos de enfrentamento e a eclosão de doenças como as oncológicas, este estudo visou investigar eventos de estresse na história prévia, a importância atribuída aos mesmos e a avaliação de superação ou não das situações relatadas por mulheres com câncer, comparativamente a mulheres sem câncer.

\section{Método}

\section{Participantes}

Fizeram parte deste estudo dois grupos de mulheres na faixa etária de 30 a 60 anos de idade. Trinta mulheres tinham diagnóstico de cânceres de mama, útero e ovários em estadiamentos (gravidade da doença) de I a IV, cujos diagnósticos foram realizados entre um a 12 meses antes da realização da entrevista para o estudo (Grupo A). As demais 30 mulheres haviam realizado exames preventivos para câncer de mama (mamografia), de útero (Exame de Papanicolau) e de ovários (exame clínico ginecológico) nos últimos 12 meses (antes da data da entrevista para o estudo), com resultados negativos para câncer e sem registro de outras doenças graves (Grupo B).

\section{Procedimento}

Os dados foram coletados no Centro Regional de Oncologia do Hospital Manoel de Abreu, na Seção de Orientação e Prevenção do Câncer da Secretaria de Saúde do Município e em Núcleos de Saúde de Bauru (SP).

Após aprovação do projeto pelas comissões de ética da Universidade Estadual Paulista e das instituições envolvidas, foram realizadas consultas aos prontuários. Dados clínico-médicos foram obtidos por meio da análise desses prontuários, com informes sobre diagnóstico e estadiamento da doença (Grupo A) e resultados de exames preventivos e/ ou informes médicos acerca da inexistência de cânceres ou outras doenças graves nas componentes do Grupo B. Foram selecionadas 30 mulheres com diagnósticos de câncer e 30 mulheres sem câncer, partindo-se das que realizaram exames ou fizeram consultas médicas mais recentemente, até atingir o número necessário de participantes, de acordo com os critérios eleitos. As mulheres selecionadas foram contatadas pessoalmente ou por telefone por profissionais das instituições e convidadas a participar. Foram informadas sobre os objetivos, responsabilidades, procedimentos e utilização dos resultados da pesquisa; nenhuma se recusou a participar e todas assinaram o Termo de Consentimento Livre e Esclarecido.

As participantes de ambos os grupos foram entrevistadas individualmente, utilizando-se um roteiro de entrevista semiestruturada para averiguação da história de eventos de estresse e de enfrentamento nas áreas "familiar", "social/ trabalho" e "saúde", ocorridos no período de um a 10 anos antes do diagnóstico da doença (Grupo A) e de um a 10 anos antes da data da entrevista (Grupo B). A cada evento de estresse relatado, as participantes de ambos os grupos foram solicitadas a avaliar se esse evento tinha sido muito importante em sua vida, relativamente importante ou pouco importante; o modo como o enfrentou (resolvendo a situação concreta; lidando com os sentimentos e emoções; mudando a maneira de encarar a situação/problema; evitando ou fugindo o máximo possível da situação, com muito sofrimento e sem ver solução, não conseguindo agir; evitando parcialmente o problema, mas acreditando ter solução), além de sua avaliação sobre ter ou não conseguido superar os problemas apontados.

As respostas das participantes foram anotadas pela entrevistadora na presença das entrevistadas. Os dados obtidos foram organizados em dois grandes grupos: dados clínico-médicos e dados da história de eventos de estresse e enfrentamento nas áreas das relações familiares, sociais e de trabalho e de saúde.

Os resultados foram analisados quantitativamente após a categorização das respostas às questões abertas e, de acordo com os objetivos do estudo, foram submetidos à análise estatística por meio do teste Qui-Quadrado (Barbetta, 2001) para análise de dados não-paramétricos. As diferenças foram consideradas estatisticamente significativas no caso de valores de $\mathrm{p} \leq 0,05$ e com tendência de significância estatística quando valores de $\mathrm{p} \leq 0,10$ foram obtidos.

\section{Resultados e Discussão}

A maioria das mulheres tinha idade entre 46 e 60 anos (83,5\% do GA e $57 \%$ do GB), era casada (60\% e $70 \%$ do GA e do GB, respectivamente) e tinha de dois a três filhos (57\% e 77\% do GA e do GB, respectivamente). Quanto à escolaridade, 43,5\% das mulheres do Grupo A tinham Ensino Fundamental incompleto, 29,5\% tinham Ensino Médio completo e 37\% tinham Ensino Superior completo. No GB, 20\% tinham Ensino Fundamental completo, 23,5\% tinham Ensino Médio completo e 30\% tinham Ensino Superior completo. Constatou-se maior número de filhos e maior nível de escolaridade entre as participantes do GB, sem ocorrência de diferenças estatísticas significativas.

Entre as participantes, a maioria declarou-se católica ( $80 \%$ do GA e $73 \%$ do GB) e da raça branca (73\% do GA e $90 \%$ do GB). A maior parte do GA (40\%) trabalhava com serviços gerais ou emprego doméstico e a maior parte do GB (43\%) tinha um trabalho compatível com a formação de Ensino Médio. No que se refere à incidência de câncer na família, 73\% das mulheres do GA e 63\% das mulheres do GB relataram a ocorrência de casos de câncer em familiares, sem diferença estatística entre os grupos.

Quanto aos dados clínico-médicos, das mulheres do GA, $67 \%$ apresentavam diagnóstico de câncer de mama, 20\% tinham diagnóstico de câncer de útero e 13\%, de câncer de ovário. Os resultados confirmam os obtidos por Neme (1999) 
e Neme e cols. (2003), com mulheres da mesma região do Estado de São Paulo, e do INCA (2008).

Com relação à gravidade da doença (estadio), verificou-se que $76,5 \%$ das mulheres tinham cânceres nos níveis I $(13,5 \%)$ e II $(63 \%)$, de menor gravidade, e 23,5\% tinham cânceres em estadios III $(13,5 \%)$ e IV (10\%), de maior gravidade, corroborando informações de Thuler e Mendonça (2005), acerca de melhora, nos últimos anos, quanto ao estadiamento inicial dos cânceres em algumas regiões do país. Os cânceres de útero e de ovários apresentaram maior gravidade do que os de mama entre as mulheres desse estudo. Os cânceres de útero e ovários tendem a ser identificados mais tardiamente, levando a diagnósticos de maior gravidade e prognósticos mais negativos.

Os resultados quanto ao tempo de realização do último exame preventivo para o câncer e de exames médicos de rotina (grupos A e B) indicaram que entre as mulheres do GA (considerando o período relatado pelas entrevistadas entre a realização de exames e o diagnóstico do câncer), 33\% tinham realizado exames há menos de um ano; $47 \%$, há cerca de um a dois anos; $10 \%$, há mais de cinco anos e $7 \%$ declararam nunca ter realizado exames preventivos. Portanto, entre as mulheres com câncer, $17 \%$ referiram frequência inadequada (mais de dois anos) de realização de exames médicos de rotina ou nunca os ter realizado. Entre as mulheres do GB, todas $(100 \%)$ tinham realizado exames no período de um ano antes da entrevista e declararam não possuir problemas de saúde, conforme critérios exigidos para compor esse grupo. Os resultados da realização de exames preventivos na população estudada são consistentes com aqueles obtidos quanto aos menores níveis de estadiamento da doença no GA e demonstram os benefícios da realização periódica de exames preventivos.

Com relação à história de eventos de estresse ocorridos nos 10 anos anteriores à doença oncológica $(\mathrm{GA})$ e 10 anos antes da entrevista para a pesquisa (GB), são apresentados os resultados obtidos por área de vida (saúde, social/trabalho e família) e o conjunto dos dados dessas três áreas. As quatro figuras que se seguem apresentam os dados organizados em quatro categorias de análise: percentuais de eventos de estresse relatados, percentuais de avaliação de 'muita importância' dada aos eventos relatados, percentuais dos modos de enfrentamento considerados negativos ou inefetivos (lidar com fuga ou evitação da situação estressante, considerando ser impossível sua resolução) e percentuais de participantes que afirmaram não ter ainda superado os problemas/eventos de estresse relatados em cada área pesquisada.

$\mathrm{Na}$ área da saúde (Figura 1) inseriram-se eventos de estresse ou problemas de saúde (exceto o câncer) como: doenças, gravidez indesejada/risco de aborto/aborto, problemas pessoais com álcool ou drogas lícitas ou ilícitas, internações hospitalares, outros problemas de saúde ou acidentes pessoais e problemas de saúde emocional.

Nessa área foram relatadas as seguintes ocorrências: doenças cardiovasculares ( $9 \%$ do GA; $9 \%$ do GB), doenças ginecológicas ( $20 \%$ do GA; $18 \%$ do GB), doenças ósseas e/ ou reumáticas ( $8 \%$ do GA), impossibilidade de engravidar (3,5\% do GA), aborto provocado (3,5\% do GA), risco de aborto ( $10 \%$ do GA), dependência de nicotina ( $7 \%$ do GA;
$3 \%$ do GB), doenças agudas não graves (10\% do GA; $20 \%$ do GB), problemas com parto ( $6 \%$ do GB), cirurgias eletivas $(30 \%$ do GA; $20 \%$ do GB), cirurgias de urgência (7\% do GA; $6 \%$ do GB), doenças físicas crônicas (3\% do GA), distúrbios endócrinos e gastroentestinais (7\% do GA; $3 \%$ do GB), acidentes com automóvel ou atropelamento ( $7 \%$ do $\mathrm{GA} ; 3 \%$ do GB), acidentes domésticos ( $3 \%$ do GB) e outros problemas corriqueiros de saúde ( $3 \%$ do GA; $12 \%$ do GB). Quanto a problemas de saúde emocional, foram relatados: transtornos afetivos com manifestação psicossomática (8\% do GA), depressão (19\% do GA), depressão com tentativa de suicídio ( $3 \%$ do GA), estresse emocional/estresse ocupacional ( $10 \%$ do GA; $3 \%$ do GB) e transtorno do pânico ( $6 \%$ do GA). Dessa forma, $46 \%$ de problemas emocionais ocorreram no GA e apenas $3 \%$ no GB $(\mathrm{p} \leq 0,05)$.

Os principais recursos utilizados para a superação dos problemas de saúde, relatados pelas mulheres que alegaram os ter superado, foram: fé e religiosidade (5\% do GA; $9 \%$ do GB), desabafos, falar o que sentia e pensava ( $5 \%$ do GA), tratamento médico (15\% do GA; $27 \%$ do GB), tratamento psicológico (10\% do GA), busca de apoio de família e/ou amigos (5\% do GA; 6\% do GB), nenhum, só a passagem do tempo ( $3 \%$ do GA), força de vontade ( $10 \%$ do GA), mudar a forma de viver/pensar ( $3 \%$ do GA), envolvimento com o trabalho (3\% do GA; 3\% do GB) e melhorar, sarar, resolver o problema, obter resultados positivos ( $3 \%$ do GA; $15 \%$ do GB. Não foram encontradas diferenças significativas entre os grupos.

Entre as entrevistadas, $35,5 \%$ do GA e $48 \%$ do GB consideraram ter superado completamente os problemas de saúde relatados, sendo que $17 \%$ das mulheres do GA e $7 \%$ do GB consideraram não os ter ainda superado.

$\mathrm{Na}$ área das relações sociais e de trabalho (Figura 2) foram inseridos problemas de relacionamento social/isolamento/ solidão/afastamento de amigos ou parentes, mudança de emprego/desemprego/necessidade de começar a trabalhar, dificuldades financeiras, problemas pessoais ou familiares com a justiça/processo ou situações similares, problemas com chefia/colegas de trabalho/vizinhos e problemas judiciais.

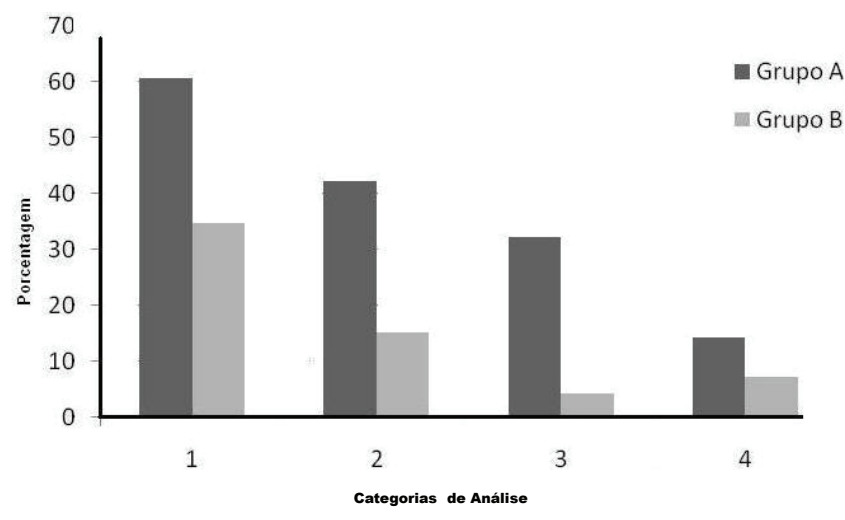

Figura 1. Estresse e enfrentamento nos 10 últimos anos na área da saúde (1. Eventos de estresse relatados - sem diferença estatística significativa; 2. Avaliação de muita importância aos eventos relatados $-\mathrm{p} \leq 0,10 ; 3$. Lidou com fuga e evitação $-\mathrm{p} \leq 0,05 ; 4$. Não superou os problemas referidos nesta área $-\mathrm{p} \leq 0,05)$. 


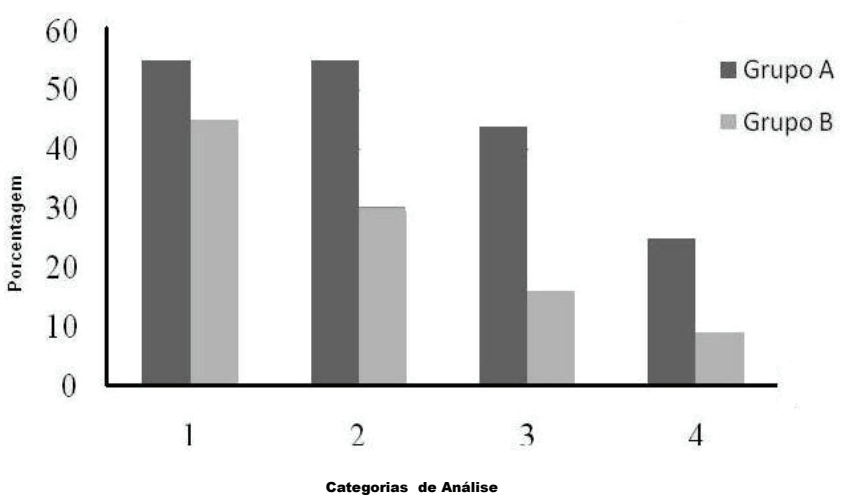

Figura 2. Estresse e enfrentamento nos 10 últimos anos na área social/ trabalho (1. Eventos de estresse relatados - sem diferença estatística significativa; 2 . Avaliação de muita importância aos eventos relatados $-\mathrm{p} \leq$ $0.10 ; 3$. Lidou com fuga e evitação $-\mathrm{p} \leq 0,05 ; 4$. Não superou os problemas referidos nesta área $-\mathrm{p} \leq 0,5)$.

Nessa área foram relatadas as seguintes ocorrências: problemas de relacionamento social, isolamento, solidão, afastamento de amigos/parentes ( $27 \%$ do GA; $10 \%$ do GB); mudança de emprego, desemprego, perda de emprego e /ou necessidade de começar a trabalhar $(27 \%$ do GA; $20 \%$ do GB); dificuldades ou problemas financeiros ( $20 \%$ do GA; $27 \%$ do GB); problemas pessoais ou familiares com a justiça (divórcios, aposentadorias, queixas etc.), processos ou situações similares (23\% do GA; $27 \%$ do GB); problemas com chefias, colegas de trabalho e/ou vizinhos (20\% do GA; $7 \%$ do GB); outros problemas de relacionamento social ou de trabalho: falta de pagamento do salário e insatisfação com colegas de trabalho ( $6 \%$ do GB).

Vários foram os fatores apontados pelas entrevistadas como fundamentais para a superação dos problemas relatados nessa área, mas a maioria $(11,5 \%)$ referiu-se à importância da fé e religiosidade, seguida de apoio familiar e de amigos $(10 \%)$, poder resolver o problema $(9 \%)$, mudar a forma de vida (6\%), tratamento psicológico (3\%), passagem do tempo (3\%) e envolvimento com o trabalho (3\%).

Quanto à consideração, pelas participantes, sobre terem ou não superado os eventos relatados na área social/traba1ho, $23,5 \%$ das mulheres do GA e $47 \%$ do GB avaliaram ter superado os problemas (com ou sem sequelas). Nos grupos A e B, $25 \%$ e $9 \%$, respectivamente, das mulheres avaliaram não ter ainda superado os problemas referidos nessa área.

$\mathrm{Na}$ área familiar (Figura 3) foram pesquisados eventos de estresse relacionados a conflitos conjugais, separações, divórcios; desentendimentos ou brigas com filhos/pais/ irmãos; perdas por morte de filhos/pais/parentes próximos; problemas de filhos/familiares com uso de drogas lícitas ou ilícitas; doenças ou acidentes graves.

As participantes relataram as seguintes ocorrências: conflitos conjugais ( $10 \%$ do GA; $6,5 \%$ do GB); separações e/ ou divórcios ( $3 \%$ do GA; $22,5 \%$ do GB); desentendimentos ou conflitos com filhos, pais ou irmãos (57\% do GA; $37 \%$ do GB); perdas por morte de filhos, pais ou parentes próximos (50\% do GA; $53 \%$ do GB); problemas devido ao uso de álcool ou drogas por filhos, cônjuge ou familiares $(30 \%$ do GA; $16 \%$ do GB); doenças ou acidentes graves sofridos por familiares ( $63 \%$ do GA; $50 \%$ do GB); outros eventos de

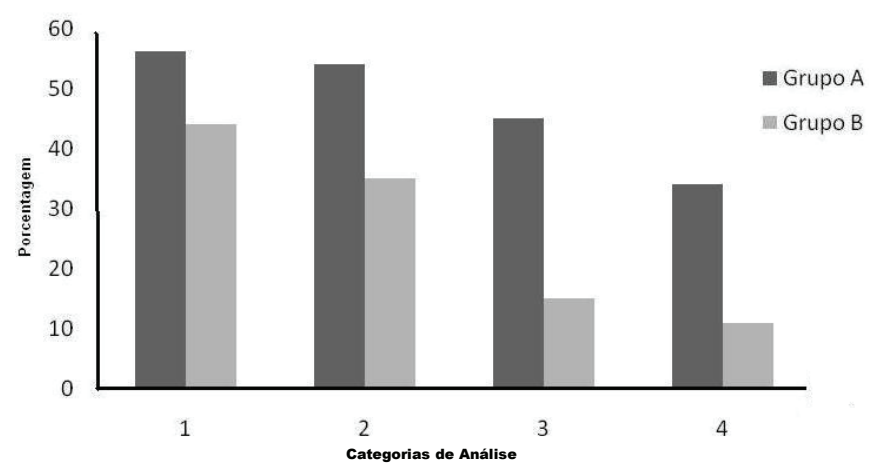

Figura 3. Estresse e enfrentamento nos 10 últimos anos na área familiar (1. Eventos de estresse relatados - sem diferença estatística significativa; 2. Avaliação de muita importância aos eventos relatados $-\mathrm{p} \leq 0,10 ; 3$. Lidou com fuga e evitação $-\mathrm{p} \leq 0,05 ; 4$. Não superou os problemas referidos nesta área $-\mathrm{p} \leq 0,5)$.

estresse na área familiar, como contrariedades com decisões de filhos (10\% do GA; $3 \%$ do GB) e ter se tornado cuidador principal de familiar doente ( $3 \%$ do $\mathrm{GA} ; 3 \%$ do GB); prisão de filhos ou familiares ( $3 \%$ do GA).

Os aspectos apontados como fundamentais para a superação dos problemas ocorridos nessa área foram: fé/religiosidade (11\% do GA; $29 \%$ do GB); desabafar, tratamento médico ( $7 \%$ do GB); tratamento psicológico ( $7 \%$ do GA; $5 \%$ do GB); apoio da família e/ou amigos ( $8 \%$ do GA; $22 \%$ do GB); nada, só a passagem do tempo ( $11 \%$ do GA); mudar a forma de vida, o modo de pensar ( $3 \%$ do GA); envolvimento com o trabalho (5\% do GB); resolver o problema, obter resultados positivos ( $3 \%$ do GA; $13 \%$ do GB).

Quanto a terem ou não superado os problemas apresentados na área das relações familiares, no grupo A, 34\% das participantes consideraram não ter ainda superado essas ocorrências e $11 \%$ avaliaram já as ter superado com ou sem sequelas.

Considerando-se a somatória dos resultados obtidos em cada uma das três áreas pesquisadas no GA e no GB, a Figura 4 apresenta os percentuais quanto ao número de eventos estressantes, à avaliação de 'muita importância' aos eventos estressantes, ao enfrentamento dos eventos relatados com fuga e evitação e à avaliação de não superação das situações de estresse referidas, indicando-se o valor de p em cada uma dessas categorias de análise.

Comparando-se a frequência de eventos de estresse relatados pelas participantes dos dois grupos nas três áreas investigadas, a área das relações familiares representou maior fonte de estresse do que as demais, em frequência de eventos e na avaliação de 'maior importância' aos eventos relatados, seguida pela área da saúde e social/trabalho. Foi também nessa área que se obteve diferença estatística significativa entre os grupos $(\mathrm{p}=0,001)$, mostrando que as mulheres do GA consideraram, muito mais que as do GB, não terem superado os problemas relatados. Esses resultados são similares aos encontrados na literatura (Lipp, 2003; Neme \& cols. 2003; Neuber \& cols., 2007), confirmando a área das relações familiares como fonte importante de estresse, com situações potencialmente mais patogênicas. Os problemas familiares podem se apresentar como conflitos tendentes 


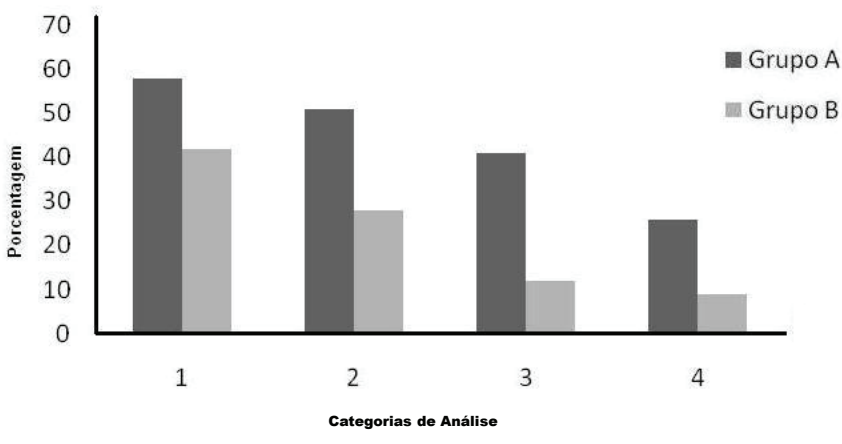

Figura 4. Somatória de respostas obtidas no GA e no GB sobre estresse e enfrentamento nas áreas da saúde, social/trabalho e relações familiares (1. Eventos de estresse relatados $-p<0,01 ; 2$. Avaliação de muita importância aos eventos relatados $-\mathrm{p}<0,01 ; 3$. Lidou com fuga e evitação $-\mathrm{p}<0,01 ; 4$. Não superou os problemas referidos nesta área $-\mathrm{p}<0,05$ ).

a se intensificar com o decorrer do tempo ou representar contrariedades permanentes.

Com relação à importância atribuída aos eventos de estresse ocorridos em sua história de vida no período considerado neste estudo, foram encontradas diferenças significativas entre os grupos A e B quanto ao número de respostas avaliadas como 'muito importante' nas três áreas investigadas (saúde social/trabalho e familiar). Pesquisas sobre o estresse psicológico indicam a relevância das avaliações cognitivo-afetivas dos estressores, que os transformam ou não em fontes internas, autogeradoras de estresse (Lipp, 2003; Lipp \& cols., 2005; Neme \& cols. 2003). As mulheres do GA também apresentaram significativamente mais relatos de problemas emocionais do que as do GB, sugerindo possíveis efeitos patogênicos do estresse prolongado ou crônico nas participantes do GA.

Outro resultado a ser considerado refere-se à área de relacionamento social e de trabalho, na qual a maioria significativa de mulheres do GA referiu ter vivido situações de afastamento social e sentimento de solidão, frequentemente mais do que as entrevistadas do GB. A solidão agrava os efeitos do estresse, favorecendo o desenvolvimento de doenças da adaptação, já que pessoas sob essas condições não dispõem de redes de apoio social para auxiliar no enfrentamento de seus problemas (Ader, 1983; Howland \& Rowland, 1990; Neme, 2005; Neuber \& cols., 2007).

Considerando que o processo de enfrentamento visa diminuir ou eliminar as fontes de estresse, reações de fuga e evitação, acompanhadas de crenças negativas sobre a possibilidade de resolver ou superar os problemas vividos e de sentimentos de desânimo ou desamparo, constituem-se modos inefetivos de lidar com o estresse. No presente estudo, as mulheres com câncer revelaram ter lidado com fuga e evitação significativamente mais que as mulheres sem câncer, com suas fontes de estresse nas três áreas pesquisadas no período de 10 anos antes do diagnóstico do câncer (GA) ou da entrevista para o estudo (GB). Fugindo ou esquivando-se de seus problemas, as mulheres com câncer não conseguiram superá-los, resolvendo-os significativamente menos do que as mulheres sem câncer $(\mathrm{p} \leq 0,05)$, tal como ocorreu com as mulheres com câncer investigadas por Neuber e cols. (2007).
Comparando-se os resultados que indicaram diferenças estatisticamente significativas e diferenças com tendências estatisticamente significativas entre os dois grupos estudados, tanto por área investigada como no cômputo geral das respostas (Figura 4), pode-se constatar que as mulheres com câncer da amostra deste estudo revelaram maior vulnerabilidade aos eventos de estresse ocorridos em sua história de vida no período de 10 anos antes da doença do que as mulheres sem câncer. As participantes sem câncer apresentaram significativamente mais respostas adaptativas, lidando mais eficientemente com os problemas vividos, conseguindo superá-los aparentemente com mais facilidade do que as participantes com câncer, as quais tenderam mais a avaliar os problemas enfrentados como muito importantes ou de difícil superação.

Tais resultados são concordantes com os encontrados na literatura, confirmando a relevância das interpretações ou avaliações cognitivo-afetivas dos eventos adversos em diferentes momentos e situações de vida, bem como a importância dos processos de enfrentamento e dos recursos pessoais para a adaptação e a superação emocional de dificuldades vividas (Alves, 1991; Neme, 2005).

Considerando-se os inevitáveis efeitos imunodepressores do estresse, pode-se sugerir que maiores dificuldades para lidar com situações estressantes e para superar as dificuldades e adversidades enfrentadas durante a vida podem favorecer a emergência de doenças relacionadas ao sistema imune, o qual funciona de forma interdependente com os sistemas neurológico, endócrino e cognitivo-emocional na gênese de muitas doenças, incluindo os cânceres (Herbert \& Cohen, 1993; Neme, 2005; Vasconcellos, 1998).

\section{Considerações Finais}

Os resultados obtidos permitem indicar a existência de relações de influência entre os modos pessoais de avaliar, enfrentar e superar situações de estresse e o adoecimento na população estudada. Sugerem que padrões mais positivos, otimistas, flexíveis e diretos de lidar com as situações e sentimentos envolvidos em situações estressantes da vida favoreceram adaptações mais saudáveis e a redução do impacto do estresse no equilíbrio psicofisiológico. Corroborando os resultados encontrados na literatura, este estudo pode contribuir para fortalecer, fundamentar e justificar a já conhecida necessidade de implementação, ampliação e adequação de programas preventivos em saúde e a elaboração de projetos psicoeducativos que visem disponibilizar conhecimentos científicos à população, especialmente no campo da saúde da mulher, atualmente exposta a alta carga de estresse psicossocial. Os programas psicoeducativos e de saúde da família devem estimular o desenvolvimento de recursos intra e interpessoais ou sociais identificados como agentes protetores do impacto do estresse e de riscos à saúde, especialmente quando se trata de uma doença grave como o câncer. Espera-se que os resultados alcançados contribuam para a solidificação de conhecimentos nas áreas de interface entre a psicologia e outras áreas da saúde como a psico-oncologia, subsidiando os profissionais na seleção de intervenções e propostas de atendimento à mulher com câncer. 


\section{Referências}

Ader, R. (1983). Developmental psychoneuroimmunology. Developmental Psychobiology, 16, 251-267.

Alves, E. (1991). Carcinogênese: fatores de risco. Jornal Brasileiro de Medicina, 61, 12-28.

Amorim, M. H. C. (1999). A enfermagem $e$ a psiconeuroimunologia no câncer de mama. Tese de Doutorado, Universidade Federal do Rio de Janeiro, Rio de Janeiro.

Barbetta, P. A. (2001). Estatística aplicada às ciências sociais (4 ${ }^{\mathrm{a}}$ ed.). Florianópolis: EdUFSC.

Bauer, M. E, Gauer G. J., \& Nardi N. B. (1993). Depressão maior e atividade do sistema imunológico. Revista da Associação Brasileira de Psiquiatria (ABP) - Associação de Psiquiatria da América Latina (APAL), 15, 87-94.

Bernardo B. C., Lorenzato F. R. B., Figueiroa J. N., \& Kitoko P. M. (2007). Disfunção sexual em pacientes com câncer do colo uterino avançado submetidas à radioterapia exclusiva. Revista Brasileira de Ginecologia e Obstetrícia, 29, 85-90.

Calais, S. L. (2003) Diferenças entre homens e mulheres na vulnerabilidade ao stress. Em M. E. N. Lipp (Org.), Mecanismos neuropsicofisiológicos do stress: teoria e aplicaçôes clinicas (pp. 87-89) ). São Paulo: Casa do Psicólogo.

Calais, S. L., Andrade, L. M. B., \& Lipp, M. E. N. (2007). Stress entre calouros e veteranos de jornalismo. Estudos de Psicologia (Campinas), 24, 69-77.

Cantinelli, F. S., Camacho, R. S., Oren, S., Gonsales, B. K., Braguittoni, E., \& Rennó JR, J. (2006). A oncopsiquiatria no câncer de mama - considerações a respeito de questões do feminino. Revista de Psiquiatria Clínica, 33, 134-133.

Carleial, B. N. (1981). Uma hipótese sobre a origem e o tratamento do câncer. Psicologia em Curso, 6, 21-27.

Carvalho, M. M. M. J. (1994). Introdução à psiconcologia. Campinas: Editorial Psi.

Carver, C. S., \& Scheier, M. F. (1994). Situational coping and coping dispositions in a stressful transaction. Journal of Personality and Social Psychology (Washington), 66, 184-195.

De Moor, J. S., de Moor, C. A., Basen-Engquist, K., Kudelka, A., Bevers, M., \& Cohen, L. (2006). Optimism, distress, health-related quality of life, and change in cancer antigen 125 among patients with ovarian cancer undergoing chemotherapy. Psychosomatic Medicine, 68, 555-562.

Dorian, B., \& Garfinkel, P. E. (1987). Stress, immunity and illness: A review. Psychological Medicine, 17, 393-407.

Duavy, L. M., Batista, F. L. R., Bessa, M. S. J., \& Santos, J. B. (2007). A percepção da mulher sobre o exame preventivo do câncer cérvico-uterino: estudo de caso. Ciência e Saúde Coletiva, 12, 733-742.

Filgueiras, M. S. T., Lisboa, A. V., Macedo, R. M., Paiva, F. G., Benfica, T. M. S., \& Vasques, V. A. (2007). Avaliação psicossomática no câncer de mama: proposta de articulação entre os níveis individual e familiar Estudos de Psicologia (Campinas), 24, 551-60.

Giovanetti, J. P. (2002). Pós-modernidade e o vazio existencial. Em D. S. P. Castro, D. D Pokladek, F. P. Ázar, J. D. Piccino \& R. S. Josgrilberg (Orgs.), Existência e Saúde (pp. 91-100). São Bernardo do Campo: FENPEC/ UMESP - SOBRPHE.

Herbert, T. B., \& Cohen, S. (1993) Stress and immunity in humans: A meta-analytic review. Psychosomatic Medicine, 55, 364-79.
Holland, J. C., \& Rowland, J. H. (1990). Handbook of psychooncology. New York: Oxford University.

Instituto Nacional do Câncer - INCA (2004). Controle do câncer de mama: documento de consenso. Revista Brasileira de Cancerologia, 50, 77-90.

Instituto Nacional do Câncer - INCA (2008). Epidemiologia, informação e vigilância. Retirado em 4/25/2008, de www.inca. gov.br.

Lazarus, R. S., \& Folkman, S. (1986). Estrés y procesos cognitivos. Barcelona: Martinez Roca.

Levin, T., \& Kissane, D. W. (2006). Psychooncology: The state of its development in 2006. European Journal of Psychiatry, 20, 189-197.

Lipp, M. E. N. (2001). Estresse emocional: a contribuição de estressores internos e externos. Revista de Psiquiatria Clínica, 28, 347-349.

Lipp, M. E. N. (2003). O Stress está dentro de você (5a ed.). São Paulo: Contexto.

Lipp, M. E. N. (2005). Stress e o turbilhão da raiva. São Paulo: Casa do Psicólogo.

Lipp, M. E. N. (2007). Controle do estresse e hipertensão arterial sistêmica. Revista Brasileira de Hipertensão, 14, 89-93.

Lipp, M. E. N., Frare, A., \& Santos F. U. (2007). Efeitos de variáveis psicológicas na reatividade cardiovascular em momentos de stress emocional. Estudos de Psicologia (Campinas), 24, 161167.

Lipp, M. E. N., Justo, A. P., \& Melo Gomes, T. (2006). Cardiovascular reactivity in hypertensives: Differential effect of expressing and inhibiting emotions during moments of interpersonal stress. The Spanish Journal of Psychology, 9, 154-161.

Lipp, M. E. N., Pereira, M. B., \& Sadir, M. A. (2005). Crenças irracionais como fontes internas de stress emocional. Revista Brasileira de Terapias Cognitivas, 1, 29-34.

Maluf, M. F. M., Mori, L. J., \& Barros, A. C. S. D. (2005). O impacto psicológico do câncer de mama. Revista Brasileira de Cancerologia, 51, 149-154.

Marszal-Wisniewska, M., \& Zakrzewska, T. (2004). Temperament and defensive coping styles as risk factors in breast cancer. Polish Psychological Bulletin, 35, 15-23.

Martins, L. F. L., Thuler, L. C. S., \& Valente, J. G. (2005). Cobertura do exame de papanicolau no Brasil: uma revisão sistemática da literatura. Revista Brasileira de Ginecologia e Obstetricia, 27, 485-92.

Moreira, M. S. (1994). Psicologia e câncer. Jornal Brasileiro de Medicina, 66, 149-161.

Müller, M. C., Hoffmann, F. S., \& Fleck, P. A. (2006). A vivência do câncer de mama e a imagem corporal na mulher contemporânea. Em B. S. G. Werlang \& M. S. Oliveira (Orgs.), Temas em psicologia clínica (pp. 203-208). São Paulo: Casa do Psicólogo.

Neme, C. M. B. (1999). Enfrentamento do câncer: ganhos terapêuticos em psicoterapia num serviço de psiconcologia em Hospital Geral. Tese de Doutorado, Pontifícia Universidade Católica, Campinas.

Neme, C. M. B. (2005). Ganhos terapêuticos com psicoterapia breve em serviço de psico-oncologia hospitalar. Em C. P. Simon, L. L. Melo-Silva \& M. A. S. Santos. Formação em psicologia: Desafios da diversidade na pesquisa e na prática (pp. 39-66). São Paulo: Vetor. 
Neme, C. M. B., \& Kato, S. (2003). Mulheres com câncer de mama: crenças sobre a doença e temores quanto ao tratamento. Em C. M. B. Neme \& O. M. P. R. Rodrigues (Orgs.), Psicologia da saúde: Perspectivas interdisciplinares (pp. 125-148). São Carlos: Rima.

Neme, C. M. B., Soliva, S. N., \& Ribeiro, E. J. (2003). História prévia de eventos de estresse e câncer de mama, útero e ovário. Em C. M. B. Neme \& O. M. P. R. Rodrigues (Orgs.), Psicologia da saúde: perspectivas interdisciplinares (pp. 95-124). São Carlos: Rima.

Neuber, L. M. B., Neme, C. M. B., Rodrigues, J. R. P., Pessoa, E. C., Kamiya, C. P., Vespoli, H. M. L., \& Uemura, G. (2007). Aspectos psicossociais e afetivo-conjugais em mulheres com e sem câncer da mama. Revista Brasileira de Mastologia, 17,156-162.

Nunes, S. O. V., Reiche, E. M. V., Morimoto, H. K., Matsuo, T., Itano, E. N., Xavier, E. C. D., Vieira, V. R., Menoli, A. V., Silva, S. S., Costa, F. B., Reiche, F. V., Silva, F. L. V., \& Kaminami, M. S. (2002). Immune and hormonal activity in adults suffering from depression. Brazilian Journal of Medical and Biological Research, 35, 581-587.

Okamura, M., Yamawaki, S., Akechi, T., Taniguchi, K., \& Uchitomi, Y. (2005). Psychiatric disorders following first breast câncer recurrence: Prevalence, associated factors and relationship to quality of life. Japanise Journal of Clinical Oncology, 35, 302-309.

Rowland, J. H. (1990). Intrapersonal resources: Coping. Em J. C. Holland \& J. H. Rowland (Eds.), Handbook of psychooncology. New York: Oxford University.
Selye, H. A. (1976). The stress of life. New York: McGraw-Hill. Solomon, G. F. (1993). Whither psychoneuroimmunology? A new era of immunology of psychosomatic medicine, and of neuroscience. Brain, Behavior and Immunity, 7, 352-366.

Temoshok, L. (1987). Personality, coping style, emotion and cancer: Towards an integrative model. Cancer Surveys, 6, 545-567.

Thuler, L. C. S., \& Mendonça, G. A. (2005). Estadiamento inicial dos casos de câncer de mama e colo do útero em mulheres brasileiras. Revista Brasileira Ginecologia Obstetrícia, 27, 656-660.

Trufelli, D. C., Miranda, V. C., Santos, M. B. B., Fraile, N. M. P., Pecoroni, P. G., Gonzaga, S. F. R., Riechelmann, R., Kaliks, R., \& Del Giglio, A. (2008). Análise do atraso no diagnóstico e tratamento do câncer de mama em um hospital público. Revista da Associação Médica Brasileira, 54, 72-76.

Vasconcellos, E. G. (1998). Tópicos de psiconeuroimunologia. São Paulo: Editorial Ipê.

Venâncio, J. L. (2004). Importância da atuação do psicólogo no tratamento de mulheres com câncer de mama. Revista Brasileira de Cancerologia, 50, 55-56.
Recebido em 01.09.08

Primeira decisão editorial em 11.01.10

Versão final em 21.09.10

Aceito em 21.09.10 